\title{
Influence of interfaces reflectivity for central thickness measurement of a contact lens by low coherence interferometry
}

\author{
I. Verrier \\ isabelle.verrier@univ-st-etienne.fr \\ C. Veillas \\ T. Lépine
}

\author{
Laboratoire Hubert Curien (UMR 5516 CNRS), Université de Lyon, Université Jean Monnet, 42000 \\ Saint-Etienne, France \\ Laboratoire Hubert Curien (UMR 5516 CNRS), Université de Lyon, Université Jean Monnet, 42000 \\ Saint-Etienne, France \\ Laboratoire Hubert Curien (UMR 5516 CNRS), Université de Lyon, Université Jean Monnet, 42000 \\ Saint-Etienne, France \\ Institut d'Optique Rhône-Alpes, 18 rue Benoît Lauras, 42000 Saint-Etienne, France
}

This paper evaluates quantitatively the relative interfaces reflectivity of a soft contact lens set in air or in water by a low coherence interferometric method. The sensitivity of the set-up is then measured and the minimum reflectivity is estimated to be as low as $0.02 \%$. Measurement of the central thickness of the lens, even when it is immersed in water, is then possible using a new supercontinuum source. [DOI: http://dx.doi.org/10.2971/jeos.2013.13006]

Keywords: Low coherence interferometry, optical instrument, contact lens

\section{INTRODUCTION}

Optical techniques are more and more used in the field of biological analysis because they are non-invasive and provide new information about tissues or objects used in medicine. In ophthalmology, for example the contact lenses properties are widely studied in order to evaluate their effect on patients with the aim to estimate their efficiency and to judge of their harmlessness. Among these characteristics, the central thickness of hydrogel contact lenses (soft) is an important parameter for visual acuity and comfort [1].

Several methods are leading to the measurement of contact lenses thickness. Among optical techniques, Optical Coherent Tomography (OCT) $[2,3]$ is now growing popular in the field of 3D biological imaging. Time Domain (TD)-OCT is a low coherence interferometric method in which the reference mirror and the object are moved to scan the entire volume of the object. Signal recording could then be quite long if high resolution is needed. Fourier Domain (FD)-OCT partially avoids this drawback by direct analysis of spectral component of the interferometric signal and reconstruction by a numerical Fourier Transform [4]. FD-OCT also named Spectral Domain (SD)-OCT is now used for several applications like cornea imaging and can be coupled to supercontinuum sources with very broadband spectral width [5]. High-resolution 3D imaging of cornea is also obtained by a particular TD-OCT method, named Full Field (FF)-OCT, that overcomes the transverse scan of the object [6]. However, for some applications, it is not useful to scan the transverse directions or to image the object in 3D when only its thickness is needed. Indeed, in particular cases, only axial scan is necessary to determine the central thickness of biological samples [7]. This is the case for the application aimed in the work presented here; moreover axial scan in our set-up is suppressed by use of a specific correlator named SISAM (Interferential Spectrometer by Selection of Amplitude Modulation) that leads directly to thickness measurement without any moving component [8].

Few years ago, we have determined the central thickness of contact lenses set in air by a low coherence interferometry setup. More recently, by the same method we have demonstrated the possibility to detect buried interfaces due to surgery in rabbit cornea [9]. This detection ability is strongly linked to the interfaces reflectivity and then to the surrounding medium of the sample. The present work aims to measure the relative interfaces reflectivity of a contact lens immersed in different surrounding media in order to determine the limit of detection of our system. Indeed, the sensitivity of the set-up is a key to improve the performances of our measurements.

\section{PRINCIPLE}

The principle of our work lies on the amplitude correlation of the electromagnetic fields issued from the reference and the measurement arms of a Mach-Zehnder interferometer. The new source associated to the interferometer has a very broadband wavelength width from 420 to $2200 \mathrm{~nm}$. The supercontinuum is generated in a micro-structured fiber pumped by a pulsed laser chip at $30 \mathrm{kHz}$ frequency rate (LEUKOS). The particularity of our set up is the combination of the fields by a SISAM correlator at the end of the interferometer, which avoids the modulation of the reference optical path [8]. This correlator is constituted of two diffraction gratings (one in each arm), a beam splitter, an imaging lens and a 2D CCD camera (Figure 1). It acts as a variable delay line across the 


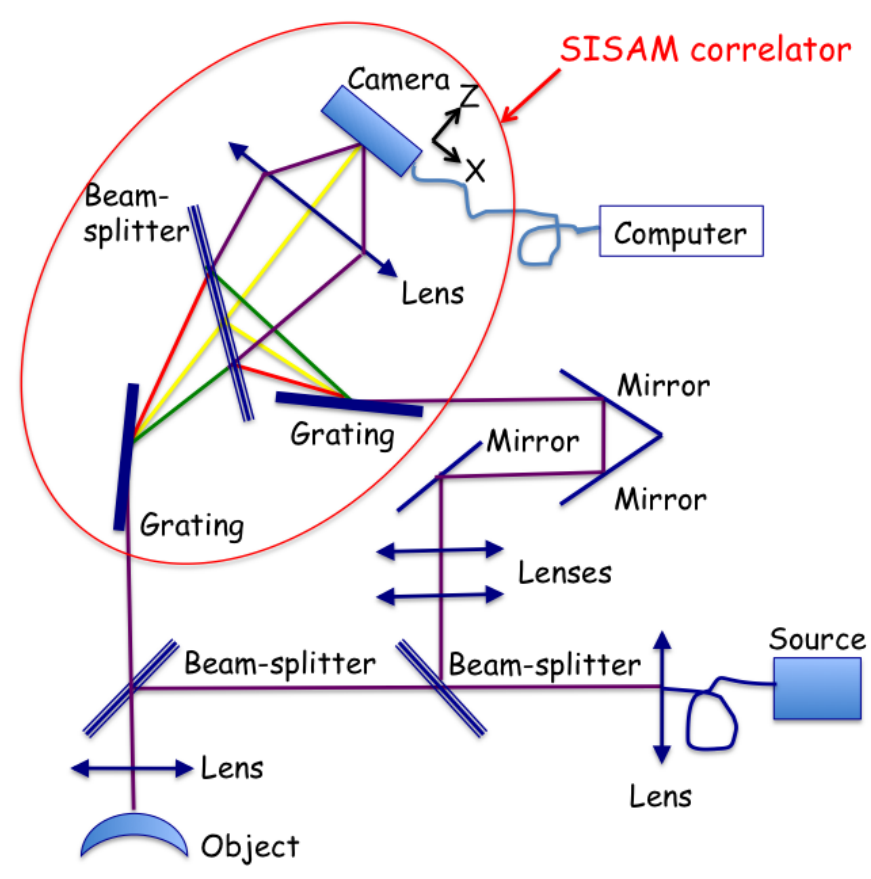

FIC. 1 Scheme of the Mach-Zehnder interferometer with the correlator.

transverse beam in the $(X, Z)$ plane and leads to a retardation of the reference and the measurement fields. Consequently, from a single image of the $2 \mathrm{D}-\mathrm{CCD}$, the correlation signal is obtained in real time. The X-axis is coding the depth of the measured sample while the Y-axis is representative from the spatial intensity distribution of the probe beam.

For samples like contact lenses (with central thickness e and group refractive index $n$ ), the intensity at the interferometer output is given by [8]:

$$
\begin{aligned}
E_{c}(X)= & E_{0}+E_{1}(X) \operatorname{sinc}\left(\pi \Delta v\left(\frac{\Delta l}{c}+Q X\right)\right) \\
& +E_{2}(X) \operatorname{sinc}\left(\pi \Delta v\left(\frac{\Delta l+2 n e}{c}+Q X\right)\right)
\end{aligned}
$$

$\Delta v$ is the source spectral bandwidth and $\Delta l=l_{T}-l_{R}$ is the difference of the reference and measurement paths in air. $X$ is the horizontal coordinate perpendicular to the grating lines (Figure 1) in the detector plane and $\mathrm{Q}$ is the SISAM scale factor depending on the central wavelength, on the incident angle on each grating and on its lines number per millimeter. $E_{0}$ is a continuous background. $E_{1}(X)$ and $E_{2}(X)$ are functions that modulate the $\operatorname{sinc}(f(X))$ envelops peaks which amplitudes are proportional respectively to $\left(R_{1}\right)^{1 / 2}$ and $\left(R_{2}\right)^{1 / 2} T_{1}$ where $R_{i}$ is the intensity reflection coefficient for the interface $\mathrm{i}(=1$ or 2$)$ and $\mathrm{T}_{1}$ the intensity transmission coefficient for the first interface.

Depending on the refractive indices of the surrounding medium (air or water solution) and of the chosen lens $(n=1.426)$, these coefficients take the value $R_{i}=3.08 \%$ or $0.12 \%$. As very weak attenuation occurs inside the contact lens, the relative amplitude between $E_{1}(X)$ and $E_{2}(X)$ is the ratio $\left(R_{2} / R_{1}\right)^{1 / 2}$ of the amplitude reflection coefficients of the two interfaces mutiplied by the intensity transmission coefficient $\mathrm{T}_{1}$ of the first interface. This relative amplitude takes the value 0.97 in the case of the lens set in air and 0.19 in water.

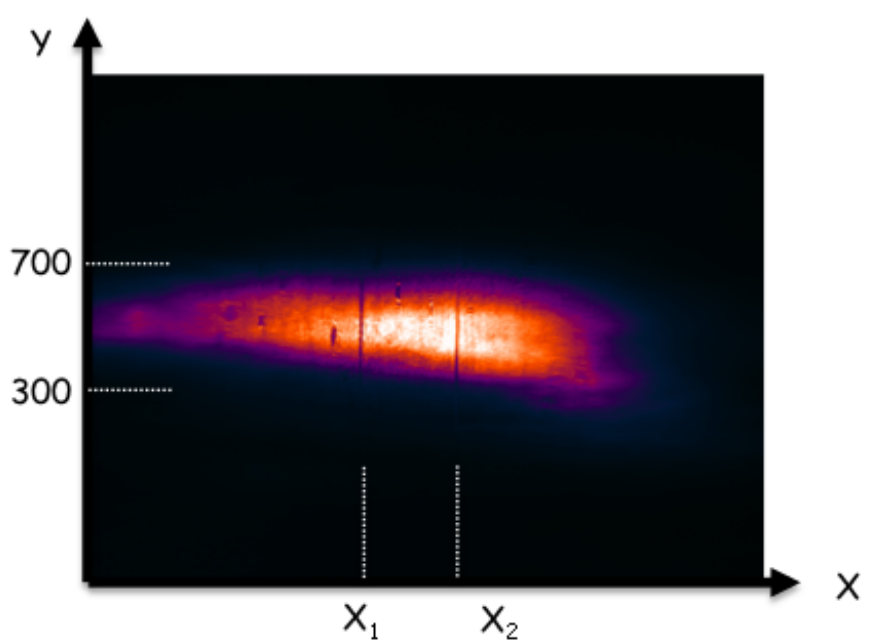

FIG. 2 Image resulting from the shift-and-add method using five images for the lens in air.

\section{MEASUREMENTS}

Among the whole recording of our video camera, five images are selected and added using shift-and-add method [9]. This technique consists in shifting the images in order to align them on one particular area. Here we choose the area corresponding to the first correlation peak (the most visible). It is only afterwards that the five shifted images are added. By this spatial adjustment on the first correlation peak, the noise due to the very small movements of the object is removed and the interference signal is enhanced leading to improvement of the Signal to Noise Ratio (SNR) proportional to the square root of the number of preselected images. The resulting image with a soft contact lens that is set in air is presented in false-color for readability (Figure 2). Two vertical areas along $X_{1}$ and $X_{2}$ exhibit peaks due to constructive interferences corresponding to the interfaces of the contact lens.

After subtraction of the background (using images corresponding to the signals of the reference and measurement arm taken separately), a region of interest (lines 300-700) corresponding to the spatial envelop of the source is selected wherein each line contains the same information about the correlation. Then filtering by a method of edge detection (Prewitt filter) and sum of the grey level on each column are realized [9]. The correlation peaks are detected at pixels $X_{1}=499$ and $X_{2}=681$ (Figure 3). They lead to an optical path difference of 182 pixels between the two echoes due to each interface of the lens. After calibration and knowing the optical index of the sample, the thickness of the lens is deduced and takes the value $91 \pm 4$ micrometers.

Furthermore the amplitudes ratio of the peaks is linked to the reflectivity of each interface. The experimental ratio is 0.81 when the lens is set in air. This is less than the forecasted value of 0.97 and will be explained in the following discussion. Moreover, the SNR of each peak is deduced from results of Figure 3 and takes the value of $21 \mathrm{~dB}$ for the first one and $20 \mathrm{~dB}$ for the second one.

Identical records and numerical treatments are realized with the same lens which second interface is immersed in water. 


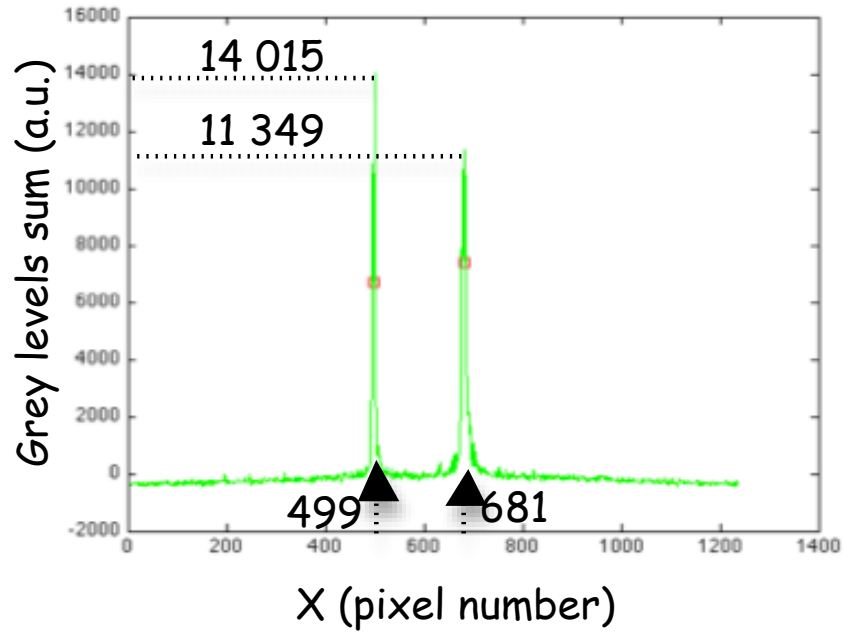

FIG. 3 Signal after numerical processing for the lens in air (5 images, lines 300-700).

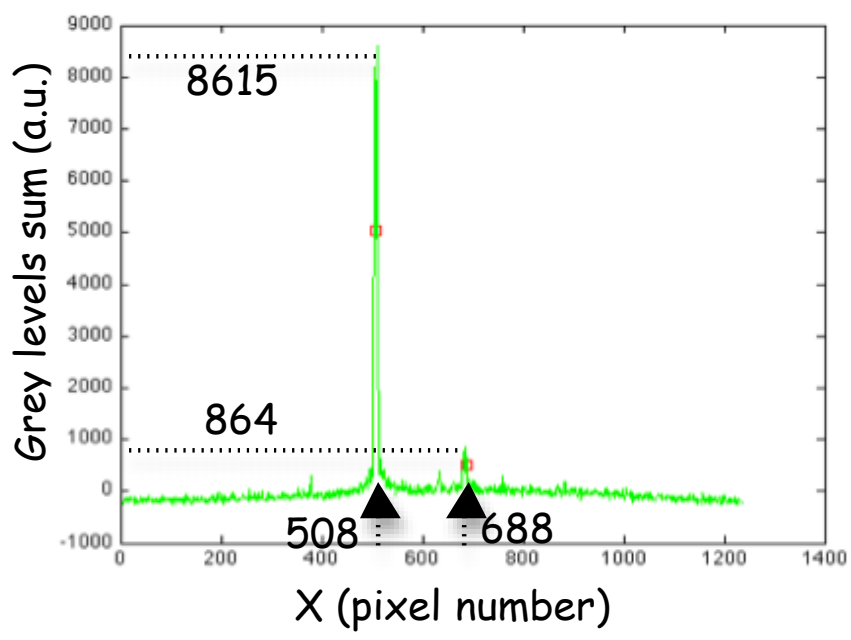

FIG. 4 Signal for the lens in water ( 5 images, lines 300-700).

Figure 4 shows the resulting signal with the correlation peaks at pixels $X^{\prime}{ }_{1}=508$ and $X_{2}^{\prime}=688$. This leads to a lens thickness of 90 micrometers. This value is the same as the one measured when the lens is set in air when considering the uncertainty measurement of 4 micrometers, which means that the lens does not swell when being immersed.

The experimental ratio of the peaks amplitude is in this case much weaker than the one obtained when the lens is in air because of the small reflectivity of the second interface. It takes the value of 0.1 that is however still less than the theoretical one of 0.19. Moreover, in this case, the SNR of each peak is $21 \mathrm{~dB}$ and $11 \mathrm{~dB}$.

Besides these measurements, the sensitivity of the set-up is also experimentally determined using a mirror as sample $\left(R_{1}=100 \%\right)$ leading to the maximum value of the SNR equal to $23 \mathrm{~dB}$.

\section{DISCUSSION}

The sensitivity of our set-up is good enough $\left(\mathrm{SNR}_{\mathrm{Max}}=23 \mathrm{~dB}\right)$ to detect the small reflectivity of the second interface when the lens is set in liquid. Indeed, choosing the minimum detectable

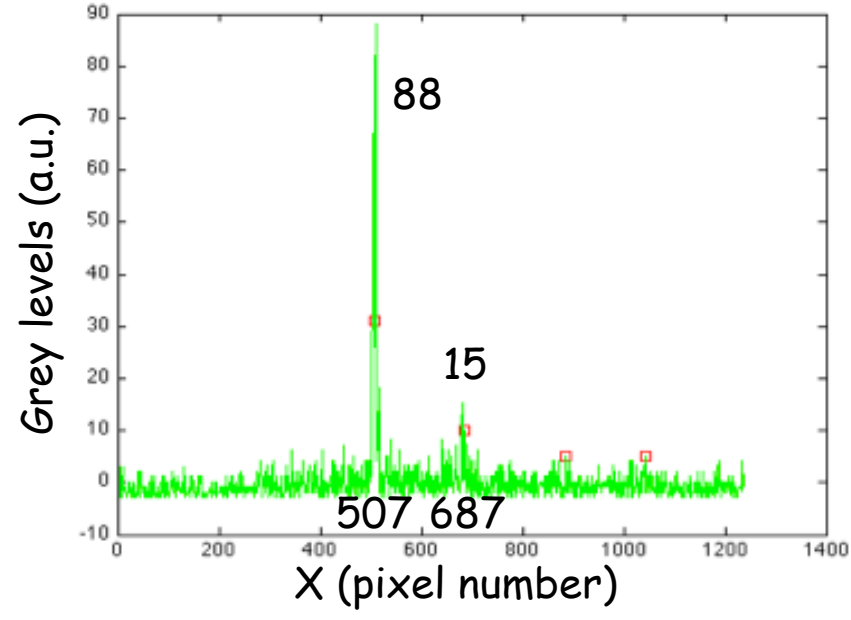

FIG. 5 Signal for the lens in water ( 5 images, line 500).

signal equal to three times the noise level, the intensity reflection coefficient takes the value of $0.02 \%$ leading to a relative index difference $\Delta n / n=0.03$.

Now, let us discuss about the amplitudes ratio of the correlation peaks and what are the factors that can increase the SNR to improve thus the sensitivity of our device. On one hand, the repetition rate of the source $(30 \mathrm{kHz}$ instead of $8 \mathrm{kHz}$ in our previous work [9]), results in a gain greater than two of the irradiance for the spectral range 420-1100 nm (response of CCD). On the other hand, besides the noise brought by the source and the detector (mainly the shot noise), the values of different parameters used for signal processing influence significantly the value of the SNR.

The discrepancy between theoretical peaks amplitudes ratios and their corresponding experimental values lies probably on the fact that the correlation signals are obtained by sum of the grey level on each column. Indeed, even if all the lines of the region of interest contain the same information, interference areas (correlation peaks) have not the same contrast on the whole column. A first approach to confirm this is to analyze one line only of the same files and to extract the maximum values of the peaks. An example of results (line 500 at the middle of the area first studied) obtained with the second interface of the lens immersed in water is reported in Figure 5.

For this considered line, the measurement of the lens thickness keeps the same value (90 micrometers) than the previous one (obtained with lines 300-700) and the amplitudes peaks ratio is 0.17 much closer to the theoretical value. However, the ratio value can slightly differ from the expected one according to the chosen line around the middle of the studied area. Furthermore, using only one line of the signal leads to noise increase: SNR peaks values become $16.8 \mathrm{~dB}$ and $9.3 \mathrm{~dB}$ (instead of $21 \mathrm{~dB}$ and $11 \mathrm{~dB}$ ). Moreover, it gives rise to detection of spurious correlation peaks that cannot be acceptable for measurement. Alike, for the lens set in air, the amplitudes peaks ratio is getting better (0.966) using only one line.

Considering the results obtained with 400 lines (Figure 4) and one line (Figure 5), it seems necessary to optimize the number 


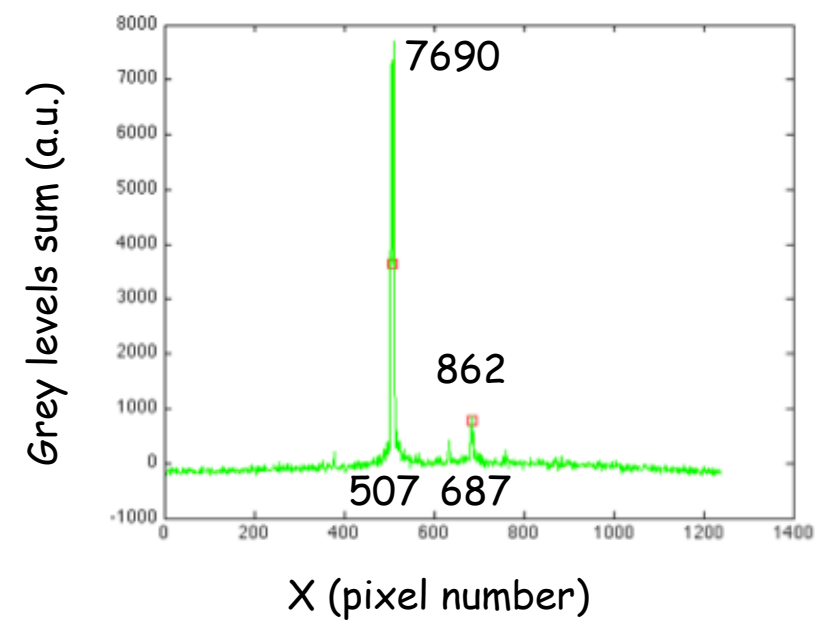

FIG. 6 Signal for the lens in water ( 5 images, lines 400-650).

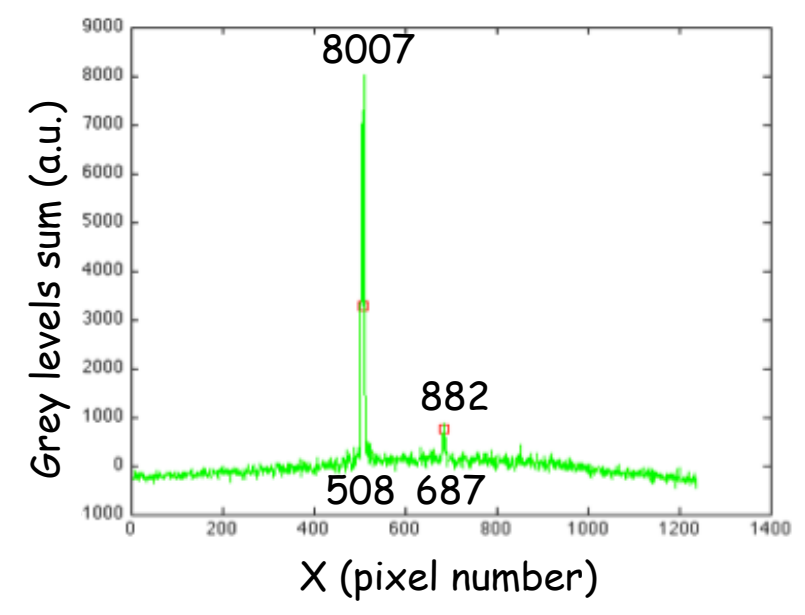

FIC. 7 Signal for the lens in water ( 1 image, lines 400-650).

of chosen lines in order to get the best SNR while not detecting unwanted maxima. After several tests, the lines 400 to 650 are taken as region of interest (Figure 6) and SNR of the two peaks are equal to $21.4 \mathrm{~dB}$ and $12.3 \mathrm{~dB}$. These values are greater than for the results of Figure 4 because only the region where the peaks are visible is taken into account.

Besides the influence of the region of interest, the number of images added after being shifted has also to be considered. Figure 7 shows that the signal obtained for one image only, presents as expected, more noise than for five images. The SNR decreases respectively to $19.3 \mathrm{~dB}$ and $10.1 \mathrm{~dB}$ (instead of $21.4 \mathrm{~dB}$ and $12.3 \mathrm{~dB}$ ).

Using more than five images does not improve significantly the SNR and on the contrary, a larger number of images could affect proper shifting. To summarize the different steps of the study of the SNR, using an appropriate number of images during the numerical processing, improves the results and the choice of the lines number for the region of interest is also a key parameter. For the particular case of the lens immersed in water, the best conditions correspond to a numerical processing with 5 images and 250 lines.

\section{CONCLUSION}

In this paper, we have demonstrated the possibility to detect interfaces and to measure thickness of a soft contact lens in a liquid medium (water) as well as in air. Our set-up is sensitive enough even if reflectivity is as low as $0.02 \%$. This is an important result for contact lens characterization that could be done in its conservation liquid without manipulation of the sample that could lead to its deterioration or contamination. Thus this study opens new outlooks for applications using biological materials in their conservation liquid and will be interesting in reflectivity evaluation. The main innovations presented here, with regard to our previous work, are the use of a more powerful light source, the thorough study of the reflectivity and of the SNR according to the different parameters used in the numerical processing. This last one has still to be improved in the future in order to keep good values for relative interfaces reflectivity and SNR simultaneously.

\section{References}

[1] J. Gispets, G. Cardona, R. Sola, C. Varon, and F. Salazar, "Central thickness of hydrogel contact lenses as a predictor of success when fitting patients with tear deficiency," Contact Lens Ct Anterior Eye 25, 89-94 (2002).

[2] J. M. Schmitt, "Optical Coherence Tomography (OCT): A review," IEEE J. Sel. Top. Quantum Electron. 5, 1205-1215 (1999).

[3] A. F. Fercher, W. Drexler, C. K. Hitzenberger, and T. Lasser, "Optical coherence tomography principles and applications," Rep. Prog. Phys. 66, 239-303 (2003).

[4] R. Leitgeb, C. K. Hitzenberger, and A. F. Fercher, "Performance of Fourier domain vs. time domain optical coherence tomography," Opt. Express 11, 889-894 (2003).

[5] M. Szkulmowski, M. Wojtkowski, T. Bajraszewski, I. Gorczynska, P. Targowski, W. Wasilewski, A. Kowalczyk, and C. Radzewicz, "Quality improvement for high resolution in vivo images by spectral domain optical coherence tomography with supercontinuum sources," Opt. Commun. 246, 569-578 (2005).

[6] G. Latour, G. Georges, L. Siozade Lamoine, C. Deumié, J. Conrath and L. Hoffart, "Human graft cornea and laser incisions imaging with micrometer scale resolution full-field optical coherence tomography," J. Biomed. Opt. 15, 56006 (2010).

[7] D-H. Kim, C-G. Song, I. K. Ilev and J. U. Kang, "Axial-scanning low coherence interferometer method for non-contact thickness measurement of biological samples," Appl. 0pt. 50, 970-974 (2011).

[8] I. Verrier, C. Veillas, and T. Lépine, "Low coherence interferometry for central thickness measurement of rigid and soft contact lenses," Opt. Express 17, 9157-9170 (2009).

[9] I. Verrier, C. Veillas, T. Lépine, F. Nguyen, G. Thuret, and P. Gain, "Interfaces detection after corneal refractive surgery by low coherence optical interferometry," Biomed. Opt. Express 1, 1460-1471 (2010). 